\title{
The role of epistasis and background genotype in the expression of heterosis
}

\author{
H. S. POONI* \& A. J. TREHARNE \\ School of Biological Sciences, University of Birmingham, Edgbaston, Birmingham, B15 2TT, U.K.
}

\begin{abstract}
The genetical control of heterosis is usually explained in terms of additive/dominance gene action and digenic interaction of segregating loci, and the contribution of epistasis categorized either as duplicate or complementary following the classical relationships observed among the Mendelian genes. In this paper we have explored the alternative relationships between the additive/ dominance/epistatic components for obtaining classical ratios and investigated their role in promoting heterosis. The study has shown that while the classical duplicate and complementary relationships supress heterosis, other relationships not only boost its magnitude but also allow more or less unrestricted expression of genotypic variation in the $F_{2}$ generation. Removal of another important restriction of no interaction between the segregating loci and the background genotype further revealed that heterosis is boosted in the presence of such interactions. However, these interactions do not affect the genetical interpretation of heterosis because their contribution is maximized only when the parental lines display dispersion of alleles at the segregating loci and dominance is unidirectional.
\end{abstract}

Keywords: epistasis, gene action, heterosis, hybrid vigour, non-allelic interaction.

\section{Introduction}

Since the pioneering work of Jinks (1954) which implicated epistasis in the expression of heterosis, attempts have been made to classify digenic interactions into two primary categories, namely duplicate and complementary (see Mather \& Jinks, 1982 for further details). While this classification makes biological sense and may have provided accurate descriptions of the type of epistasis prevailing in numerous crosses, it is unlikely to be universal because allele dispersion has to be invoked to explain the relative directions and magnitudes of the various epistatic components on many occasions (Jinks, 1983; Kearsey \& Pooni, 1992). Further, the nature of duplicate and complementary relationships is such that the double heterozygote $(A a B b)$ is expected to have the same phenotype as one of the two homozygous parents which show allelic association $(A A B B$ or $a a b b)$ and such conditions negate the expression of heterosis, at least in a dihybrid cross. An alternative explanation can be that the classical duplicate and complementary relationships do not hold between the components in general and other relationships that favour the expression of heterosis (under specific conditions) are

*Correspondence. observed more frequently (Pooni \& Jinks, 1981). Futhermore, such discrepancies in the interpretation of epistasis may also be caused by the impact of the background genotype, particularly when alleles fixed in the background interact with those that are segregating in the cross.

In the present study, we investigate both these aspects by computer simulation. Initially, we identify alternative relationships that yield known classical ratios in a dihybrid cross and/or promote heterosis in the $\mathrm{F}_{1}$ hybrid and explore their impact on the interpretation of the cause of heterosis. Next, the same relationships are screened for those cases where interactions between the background and the segregating alleles lead to the expression of heterosis. Later on, these models are extended to multilocus situations and their impact on the validity of the genetical analysis in general, and the interpretation of components of heterosis in particular, is investigated in detail.

\section{Genotypes and expectations}

Initially we consider a two locus $(A / a$ and $B / b)$ model. The expectations of the nine genotypes in the $F_{2}$ generation of a dihybrid cross are given in Table 1 . The $3 \times 3$ table allows us to visualize all kinds of comparisons for a given genetical situation. For example, we 
can compare the double heterozygote $A a B b$ along the leading diagonal and determine if the dihybrid exhibits heterosis when alleles are associated in the parents. A similar comparison along the off-diagonal determines the impact of gene dispersion on the dihybrid heterosis and comparisons of the three scores within row 1 or row 3 or column 1 or column 3 allow us to study the effects of the background alleles on the performances of monohybrids.

\section{Complementary and duplicate relationships}

Initially we look at four segregation ratios that have already been considered by Mather (1967) and Mather \& Jinks (1982). These ratios are 15:1, 12:3:1, 9:7 and $9: 3: 4$ and they represent classical duplicate, dominant, complementary and recessive epistasis, respectively. The conditions under which these ratios are obtained in biometrical genetics are (a) complete dominance, (b) unidirectional dominance and (c) complete epistasis for duplicate and complementary epistases and, in addition, unequal gene effects for the dominant and recessive epistases. For a situation where $h_{\mathrm{a}}$ and $h_{\mathrm{b}}$ are positive, the ratios obtained when certain groups of genotypes have the same phenotype are shown in Table 2.

It is apparent from Table 2 that $A A B B$ (or $a a b b$ ) and $A a B b$ must take the same phenotype for each relationship to hold true. This indicates clearly that classical duplicate and complementary epistases do not allow the dihybrid to exhibit heterosis when the parents show complete association of alleles. Similarly, no heterosis can be observed in the presence of duplicate or dominant epistasis even when parents show complete dispersion of alleles $(A A b b$ and $a a B B)$. A modest level of heterosis, however, can be observed in the presence of recessive epistasis but only when the parents also display allele dispersion.

Table 1 Expectations of the nine genotypes in the $\mathrm{F}_{2}$ generation of a dihybrid cross

\begin{tabular}{lccc}
\hline Genotype & $A A B B$ & $A a B B$ & $a a B B$ \\
Frequency & $1 / 16$ & $2 / 16$ & $1 / 16$ \\
Expectation & $m+d_{\mathrm{a}}+d_{\mathrm{b}}+i_{\mathrm{ab}}$ & $m+h_{\mathrm{a}}+d_{\mathrm{b}}+j_{\mathrm{ba}}$ & $m-d_{\mathrm{a}}+d_{\mathrm{b}}-i_{\mathrm{ab}}$ \\
Genotype & $A A B b$ & $A a B b$ & $a a B b$ \\
Frequency & $2 / 16$ & $4 / 16$ & $2 / 16$ \\
Expectation & $m+d_{\mathrm{a}}+h_{\mathrm{b}}+j_{\mathrm{ab}}$ & $m+h_{\mathrm{a}}+h_{\mathrm{b}}+l_{\mathrm{ab}}$ & $m-d_{\mathrm{a}}+h_{\mathrm{b}}-j_{\mathrm{ab}}$ \\
Genotype & $A A b b$ & $A a b b$ & $a a b b$ \\
Frequency & $1 / 16$ & $2 / 16$ & $1 / 16$ \\
Expectation & $m+d_{\mathrm{a}}-d_{\mathrm{b}}-i_{\mathrm{ab}}$ & $m+h_{\mathrm{a}}-d_{\mathrm{b}}-j_{\mathrm{ba}}$ & $m-d_{\mathrm{a}}-d_{\mathrm{b}}+i_{\mathrm{ab}}$ \\
\hline
\end{tabular}

Expectations of these genotypes are defined on the $\mathrm{F}_{\infty}$ metric of Smith \& Robson (1959) where various symbols represent the following effects. $d_{\mathrm{a}}$ and $d_{\mathrm{b}}$ : additive deviations of alleles at $A / a$ and $B / b$ loci. $h_{\mathrm{a}}$ and $h_{\mathrm{b}}$ : dominance effects of $A / a$ and $B / b$ loci. $i_{\mathrm{ab}}$ : additive $\times$ additive interaction. $j_{\mathrm{ab}}$ and $j_{\mathrm{ba}}$ : additive $\times$ dominance interaction. $l_{\mathrm{ab}}$ : dominance $\times$ dominance interaction.

Table 2 Phenotypic relationships of the various groups of genotypes in the presence of the four classical types of heterosis when dominance is positive at both the $A / a$ and $B / b$ loci

$\left.\begin{array}{lccccc}\hline \text { Genotype } & \text { Frequency } & \begin{array}{c}\text { Duplicate } \\ \text { epistasis }\end{array} & \begin{array}{c}\text { Dominant } \\ \text { epistasis }\end{array} & \begin{array}{c}\text { Complementary } \\ \text { epistasis }\end{array} & \begin{array}{c}\text { Recessive } \\ \text { epistasis }\end{array} \\ \hline A A B B & 1 / 16 \\ A A B b & 2 / 16 \\ A a B B & 2 / 16 \\ A a B b & 4 / 16 \\ A a b b & 2 / 16 \\ A A b b & 1 / 16 \\ a a B B & 1 / 16 \\ a a B b & 2 / 16\end{array}\right\} \begin{aligned} & 15 \\ & a a b b\end{aligned}$

$\uparrow$ Bracketed genotypes have the same phenotype. 


\section{Alternative relationships}

Mather (1967) defined the duplicate and complementary relationships assuming that all the epistatic components were of equal magnitude. $\mathrm{He}$ also suggested that each of the $i_{\mathrm{ab}}, j_{\mathrm{ab}}, j_{\mathrm{ba}}$ and $l_{\mathrm{ab}}$ components can take a positive, negative or zero value and the same can also be true for the dominance effects $h_{\mathrm{a}}$ and $h_{\mathrm{b}}$ as well. Using these values, we generate all $3^{6}$ (=729) combinations of $d, h, i, j$ and $l$ (see Mather, 1967 for details) and then identify those combinations that either yield various classical relationships or assist in the expression of heterosis, both positive and negative.

The numbers of combinations that gave the classical ratios are shown in Table 3. Perusal of these combinations reveals that 15:1 and 9:7 ratios are obtained from only those relationships of the dominance and epistatic parameters that have already been described by Mather (1967). The ratios of 12:3:1 and 9:3:4 are, however, given by several other combinations which incidentally assume complete or no dominance at each locus and complete or no interaction between the alleles of the two loci. It is also interesting to note that four, four and eight combinations provided 13:3, $10: 3: 3$ and $7: 5: 4$ ratios, respectively. Further details of these combinations are given in Table 4.

\section{Heterotic relationships}

It is perhaps worth pointing out that none of the above combinations leads to heterosis in the dihybrid when parents are associated for alleles at the loci under consideration. However, 90 of the 729 combinations do specify conditions where $A a B b$ is expected to perform better than $A A B B$ and $a a b b$, at least in theory. As expected, these combinations are equally divided between positive and negative heterosis and the 45 combinations giving positive heterosis are presented in Table 5.

Table 3 Numbers of combinations that give the classical ratios

\begin{tabular}{lc}
\hline Ratio & No. of combinations \\
\hline $15: 1$ & -2 \\
$13: 3$ & 4 \\
$12: 3: 1$ & 8 \\
$10: 3: 3$ & 4 \\
$9: 7$ & 2 \\
$9: 3: 4$ & 12 \\
$7: 5: 4$ & 8 \\
\hline
\end{tabular}

These comparisons show clearly that additive $\times$ additive and dominance $\times$ dominance interactions are not only essential for the expression of heterosis in the dihybrid but heterosis is maximized when $i_{\mathrm{ab}}$ and $l_{\mathrm{ab}}$ take opposing signs and dominance and dominance $\times$ dominance interaction reinforce each other. Further, it is possible to observe heterosis even

Table 4 Signs and coefficients of the eight components for the various known classical relationships

\begin{tabular}{|c|c|c|c|c|c|c|c|}
\hline Ratio & $d_{\mathrm{a}}$ and $d_{\mathrm{b}}$ & $h_{\mathrm{a}}$ & $h_{\mathrm{b}}$ & $i_{\mathrm{ab}}$ & $j_{\mathrm{ab}}$ & $j_{\mathrm{ba}}$ & $l_{\mathrm{ab}}$ \\
\hline \multirow[t]{2}{*}{$15: 1$} & +1 & +1 & +1 & -1 & -1 & -1 & -1 \\
\hline & +1 & -1 & -1 & +1 & -1 & -1 & +1 \\
\hline \multirow[t]{4}{*}{$13: 3$} & +1 & +1 & -1 & -1 & +1 & -1 & +1 \\
\hline & +1 & +1 & -1 & +1 & -1 & +1 & -1 \\
\hline & +1 & -1 & +1 & -1 & -1 & +1 & +1 \\
\hline & +1 & -1 & +1 & +1 & +1 & -1 & -1 \\
\hline \multirow[t]{8}{*}{$12: 3: 1$} & +1 & +1 & +1 & +1 & -1 & -1 & -1 \\
\hline & +1 & +1 & 0 & 0 & -1 & 0 & -1 \\
\hline & +1 & 0 & +1 & 0 & 0 & -1 & -1 \\
\hline & +1 & 0 & 0 & +1 & -1 & -1 & 0 \\
\hline & +1 & -1 & -1 & -1 & -1 & -1 & 0 \\
\hline & +1 & -1 & 0 & 0 & -1 & 0 & +1 \\
\hline & +1 & 0 & -1 & 0 & 0 & -1 & +1 \\
\hline & +1 & 0 & 0 & -1 & -1 & -1 & 0 \\
\hline \multirow[t]{4}{*}{$10: 3: 3$} & +1 & -1 & +1 & 0 & 0 & 0 & 0 \\
\hline & +1 & 0 & 0 & 0 & -1 & +1 & 0 \\
\hline & +1 & 0 & 0 & 0 & +1 & -1 & 0 \\
\hline & +1 & +1 & -1 & 0 & 0 & 0 & 0 \\
\hline \multirow[t]{2}{*}{$9: 7$} & +1 & +1 & +1 & +1 & +1 & +1 & +1 \\
\hline & +1 & -1 & -1 & -1 & +1 & +1 & -1 \\
\hline \multirow[t]{12}{*}{$9: 3: 4$} & +1 & -1 & 0 & 0 & +1 & 0 & -1 \\
\hline & +1 & -1 & +1 & -1 & -1 & +1 & -1 \\
\hline & +1 & -1 & +1 & -1 & -1 & +1 & 0 \\
\hline & +1 & -1 & +1 & +1 & +1 & -1 & 0 \\
\hline & +1 & -1 & +1 & +1 & +1 & -1 & +1 \\
\hline & +1 & 0 & -1 & 0 & 0 & +1 & -1 \\
\hline & +1 & 0 & +1 & 0 & 0 & +1 & +1 \\
\hline & +1 & +1 & -1 & -1 & +1 & -1 & -1 \\
\hline & +1 & +1 & -1 & -1 & +1 & -1 & 0 \\
\hline & +1 & +1 & -1 & +1 & -1 & +1 & 0 \\
\hline & +1 & +1 & -1 & +1 & -1 & +1 & +1 \\
\hline & +1 & +1 & 0 & 0 & +1 & 0 & +1 \\
\hline \multirow[t]{8}{*}{$7: 5: 4$} & +1 & -1 & -1 & -1 & -1 & +1 & -1 \\
\hline & +1 & -1 & -1 & -1 & +1 & -1 & -1 \\
\hline & +1 & -1 & -1 & -1 & +1 & +1 & 0 \\
\hline & +1 & -1 & -1 & -1 & +1 & +1 & +1 \\
\hline & +1 & +1 & +1 & +1 & -1 & +1 & +1 \\
\hline & +1 & +1 & +1 & +1 & +1 & -1 & +1 \\
\hline & +1 & +1 & +1 & +1 & +1 & +1 & -1 \\
\hline & +1 & +1 & +1 & +1 & +1 & +1 & 0 \\
\hline
\end{tabular}


Table 5 Signs and coefficients of the six components for the various relationships that promote heterosis

\begin{tabular}{|c|c|c|c|c|c|c|c|c|c|c|c|c|c|}
\hline Ratio & $h_{\mathrm{a}}$ & $h_{\mathrm{b}}$ & $i_{\mathrm{ab}}$ & $j_{\mathrm{ab}}$ & $j_{\mathrm{ba}}$ & $l_{\mathrm{ab}}$ & Ratio & $h_{\mathrm{a}}$ & $h_{\mathrm{b}}$ & $i_{\mathrm{ab}}$ & $j_{\mathrm{ab}}$ & $j_{\mathrm{ba}}$ & $l_{\mathrm{ab}}$ \\
\hline \multirow[t]{2}{*}{$11: 4: 1$} & 1 & 1 & -1 & -1 & -1 & 0 & \multirow[t]{2}{*}{$6: 6: 3: 1$} & 0 & 1 & -1 & 0 & -1 & 1 \\
\hline & 1 & 1 & -1 & -1 & -1 & 1 & & 1 & 0 & -1 & -1 & 0 & 1 \\
\hline \multirow[t]{4}{*}{$9: 4: 2: 1$} & 0 & 1 & -1 & -1 & 0 & 1 & \multirow[t]{2}{*}{$6: 5: 4: 1$} & \multirow[t]{2}{*}{1} & \multirow[t]{2}{*}{1} & \multirow[t]{2}{*}{0} & \multirow[t]{2}{*}{0} & \multirow[t]{2}{*}{0} & \multirow[t]{2}{*}{1} \\
\hline & 1 & 0 & -1 & 0 & -1 & 1 & & & & & & & \\
\hline & & & & & & & \multirow[t]{2}{*}{$6: 5: 2: 2: 1$} & 0 & 1 & -1 & 0 & 0 & 1 \\
\hline & & & & & & & & 1 & 0 & -1 & 0 & 0 & 1 \\
\hline \multirow[t]{3}{*}{$8: 4: 3: 1$} & 1 & 1 & -1 & 0 & 0 & 0 & & & & & & & \\
\hline & 1 & 1 & -1 & 1 & 1 & 1 & $6: 4: 3: 2: 1$ & 1 & 1 & 0 & 0 & 1 & 1 \\
\hline & & & & & & & & 1 & 1 & 0 & 1 & 0 & 1 \\
\hline \multirow[t]{3}{*}{$8: 4: 2: 1: 1$} & 1 & 1 & 0 & -1 & -1 & 1 & \multirow{3}{*}{$6: 4: 2: 2: 1: 1$} & & & & & & \\
\hline & 1 & 1 & 0 & 1 & 1 & 1 & & 1 & 1 & 0 & 1 & -1 & 1 \\
\hline & & & & & & & & 1 & 1 & 0 & -1 & 1 & 1 \\
\hline \multirow[t]{3}{*}{$8: 3: 2: 2: 1$} & 0 & 1 & -1 & 0 & 0 & 1 & \multirow{3}{*}{$6: 3: 2: 2: 2: 1$} & & & & & & \\
\hline & 1 & 0 & -1 & 1 & 0 & 1 & & 0 & 1 & -1 & 1 & 1 & 1 \\
\hline & & & & & & & & 1 & 0 & -1 & 1 & 1 & 1 \\
\hline \multirow{7}{*}{$7: 6: 2: 1$} & 0 & 1 & -1 & 0 & -1 & 1 & & 1 & 1 & -1 & 0 & 1 & 0 \\
\hline & 1 & 0 & -1 & 1 & 1 & 1 & & 1 & 1 & -1 & 0 & 1 & 1 \\
\hline & 1 & 1 & -1 & 1 & -1 & 1 & & 1 & 1 & -1 & 1 & 0 & 0 \\
\hline & 1 & 1 & -1 & -1 & 0 & 0 & & 1 & 1 & -1 & 1 & 0 & 1 \\
\hline & 1 & 1 & -1 & 0 & -1 & 0 & & & & & & & \\
\hline & 1 & 1 & -1 & -1 & 1 & 1 & \multirow[t]{2}{*}{$5: 4: 4: 2: 1$} & 0 & 1 & -1 & 1 & 0 & 1 \\
\hline & & & & & & & & 1 & 0 & -1 & 0 & 1 & 1 \\
\hline \multirow[t]{3}{*}{$7: 4: 4: 1$} & 1 & 0 & -1 & -1 & -1 & 1 & \multirow[t]{3}{*}{$4: 4: 4: 3: 1$} & 1 & 1 & -1 & 0 & 0 & 1 \\
\hline & 0 & 1 & -1 & -1 & -1 & 1 & & 1 & 1 & -1 & 1 & 1 & 0 \\
\hline & & & & & & & & 1 & 1 & 0 & -1 & 0 & 1 \\
\hline \multirow[t]{4}{*}{$7: 4: 2: 2: 1$} & 1 & 1 & -1 & -1 & 0 & 1 & & 1 & 1 & 0 & 0 & -1 & 1 \\
\hline & 1 & 1 & -1 & -1 & 1 & 0 & \multirow{3}{*}{$4: 4: 3: 2: 2: 1$} & & & & & & \\
\hline & 1 & 1 & -1 & 0 & -1 & 1 & & 0 & 1 & -1 & 1 & -1 & 1 \\
\hline & 1 & 1 & -1 & 1 & -1 & 0 & & 1 & 0 & -1 & -1 & 1 & 1 \\
\hline
\end{tabular}

$d_{\mathrm{a}}=d_{\mathrm{b}}=+1$

when true dominance is partial and either $i_{\mathrm{ab}}$ or $l_{\mathrm{ab}}$ is zero. It is also apparent that, as expected, the additive $\times$ dominance interaction does not play any part in the determination of dihybrid heterosis. Furthermore, in no case does the presence of epistasis lead to as much reduction in the phenotypic diversity as is observed in almost all the classical cases.

\section{Heterosis and gene dispersion}

Irrespective of the presence or absence of epistasis, dihybrid heterosis is likely to be detected in the presence of gene dispersion unless allele effects are markedly unequal and dominance is more or less completely ambidirectional. Therefore, unless these rather strict conditions are met, heterosis is likely to be observed in all those cases where parents show gene dispersion, and particularly when intra-allelic and interallelic effects are equal in magnitude, as in the present case.

Screening of all the 729 combinations shows that dispersion makes the dihybrid better than that of its better parent on more than 562 occasions.

\section{Background genotype}

Another source of variation which is rarely considered in the context of heterosis expression is the alleles that are fixed in the background genotype of the cross. The contribution of this source to the expression of heterosis is difficult to study because its effects are often confounded with other components. Secondly, it is not important unless there is interaction between the background genotype and the loci that are heterozygous in the cross. 
Although the present study allows an in-depth analysis of the impact of background alleles viz $\dot{a} v i z$ the dominance/interaction relationships, for convenience we have looked at only those cases where gene effects are equal and dominance is complete at either locus (e.g. $d_{\mathrm{a}}=d_{\mathrm{b}}=h_{\mathrm{a}}=h_{\mathrm{b}}=1.0$ ) and each of the four epistatic components may take coefficients of -1 , 0 and +1 . Perusal of these cases reveals that both additive $\times$ additive and additive $\times$ dominance interactions are important for the expression of heterosis in the present situation and both their positive and negative values promote positive heterosis. Further, whenever $i_{\mathrm{ab}}$ is present it makes the parental scores very similar compared to those cases where only $j_{\mathrm{ab}}$ or $j_{\mathrm{ba}}$ is significant.

Similarly, both positive and negative $i_{\mathrm{ab}}, j_{\mathrm{ab}}$ and $j_{\mathrm{ba}}$ promote negative heterosis, but only when dominance is negative too.

\section{Dominance ratio}

The dominance ratio is one of the most important parameters which is often used to discriminate between gene dispersion and overdominance as the main cause of heterosis. It is estimated as $\sqrt{H / D}$ where $H=\Sigma h^{2}$ and $D=\Sigma d^{2}$ in the absence of epistasis. Pooni (1976) showed that both additive genetic $D$ and dominance $H$ components are biased by the presence of epistasis and that the dominance ratio is affected not only by the type of epistasis but also by the distribution of alleles (dispersion/association) in the parents. In the present case, we calculate dominance ratios for all the relationships given in Tables 4 and 5 with the objective of studying the effects of various types of epistasis on dominance ratios and to identify those heterotic relationships which may give reduced or partial dominance ratios, like the ones observed in many cases (see Jinks, 1955; Kearsey \& Pooni, 1992 for further details). These dominance ratios were calculated from the estimates of $D$ and $H$ which were obtained from the $L_{1 i}+L_{2 i}$ and $L_{1 i}-L_{2 i}$ comparisons of the triple test cross (see Kearsey \& Jinks, 1968 for details) assuming linkage equilibrium and allelic dispersion/association in the tester parents. The results are summarized in Table 6.

Interaction between the background and the segregating alleles also affects the dominance ratio and the direction of the bias depends very much on the nature of alleles fixed in the background and on the type of epistasis they display. For example, suppose that locus $B / b$ is segregating and locus $A / a$ has allele ' $A$ ' fixed in the background, a triple test cross analysis of such a cross yields $D=\left(d_{\mathrm{b}}+i_{\mathrm{ab}}\right)^{2}$ and $H=\left(h_{\mathrm{b}}+j_{\mathrm{ab}}\right)^{2}$ and $\sqrt{H / D}$ from these estimates will be biased upwards or
Table 6 Simulated dominance ratios obtained for the various epistatic relationships listed in Tables 4 and 5

\begin{tabular}{lll}
\hline \multicolumn{1}{c}{ Ratio } & Associated testers & Dispersed testers \\
\hline $15: 1$ & $1.5,2.1$ & $2.0,2.0$ \\
$13: 3$ & $1.4,1.4,1.5,2.1$ & $1.1,1.1,0.5,0.5$ \\
$12: 3: 1$ & $1.5,1.5 \dagger, 1.6 \dagger, 1.4^{*}$, & $6.0,0.7 \dagger, 0.6 \dagger, 0.5^{*}$, \\
& $1.4,1.7 \dagger, 1.3 \dagger, 1.3^{*}$ & $1.1,0.3 \dagger, 1.0 \dagger, 1.0^{*}$ \\
$10: 3: 3$ & $1.4,1.4^{*}, 1.7^{*}, 1.7$ & $1.0,1.0^{*}, 1.4^{*}, 1.4$ \\
$9: 7$ & $1.8,1.0$ & $1.5,0.7$ \\
$9: 3: 4$ & $1.8 \dagger, 1.0,1.4,1.4$, & $1.5 \dagger, 0.7,1.1,1.1$, \\
& $1.5,1.6 \dagger, 1.6 \dagger, 1.3$, & $1.5,0.6 \dagger, 0.6 \dagger, 1.0$, \\
& $1.3,1.0,1.0,1.7 \dagger$ & $1.0,1.5,1.5,0.3 \dagger$ \\
$7: 5: 4$ & $1.6,1.3,1.8,1.3$, & $0.3,0.4,0.7,0.4$, \\
& $1.8,1.4,1.4,2.0$ & $0.7,0.5,0.5,0.9$ \\
$11: 4: 1$ & $1.5,1.3$ & $0.2,2.7$ \\
$9: 4: 2: 1$ & $1.3 \dagger, 1.1 \dagger$ & $2.7 \dagger, 0.8 \dagger$ \\
$8: 4: 3: 1$ & $1.1,1.0$ & $0.8,0.0$ \\
$8: 4: 2: 1: 1$ & $1.0,1.0$ & $0.0,1.0$ \\
$8: 3: 2: 2: 1$ & $1.0 \dagger, 1.0 \dagger$ & $1.0 \dagger, 1.0 \dagger$ \\
$7: 6: 2: 1$ & $1.0 \dagger, 0.5 \dagger, 0.7$, & $1.0 \dagger, 2.0 \dagger, 6.0$, \\
& $0.5,0.7,0.5$ & $1.0,2.0,1.0$ \\
$7: 4: 4: 1$ & $0.5 \dagger, 0.5 \dagger$ & $1.0 \dagger, 1.0 \dagger$ \\
$7: 4: 2: 2: 1$ & $0.7,1.0,0.0,0.0$ & $2.0,1.0,0.5,0.5$ \\
$6: 6: 3: 1$ & $1.0 \dagger, 1.0 \dagger$ & $1.0 \dagger, 1.0 \dagger$ \\
$6: 5: 4: 1$ & 1.0 & 1.0 \\
$6: 5: 2: 2: 1$ & $1.0 \dagger, 0.7 \dagger$ & $0.8 \dagger, 1.5 \dagger$ \\
$6: 4: 3: 2: 1$ & $0.8,0.8$ & $1.2,1.2$ \\
$6: 4: 2: 2: 1: 1$ & $0.7,1.0$ & $1.5,0.8$ \\
$6: 3: 2: 2: 2: 1$ & $1.0 \dagger, 0.7 \dagger, 0.8$, & $0.8 \dagger, 1.5 \dagger, 1.2$, \\
$5: 4: 4: 2: 1$ & $0.8,0.7,1.0$ & $1.2,1.5,0.8$ \\
$4: 4: 4: 3: 1$ & $0.7,0.5,0.7 \dagger$ & $2.1 \dagger, 2.1 \dagger$ \\
$4: 4: 3: 2: 2: 1$ & $0.5 \dagger, 0.7 \dagger$ & $0.7,0.5,2.1,2.1$ \\
\hline & & $0.5 \dagger, 0.7 \dagger$ \\
\hline & & \\
& & \\
& &
\end{tabular}

True dominance ratio is zero and 0.5 for cases marked * and $\dagger$ respectively. In the remaining cases $\sqrt{H / D}=1.0$.

downwards, depending upon the directions and magnitudes of $i_{\mathrm{ab}}$ and $j_{\mathrm{ab}}$. When allele ' $a$ ' is fixed in the background the expectations of $D$ and $H$ become $\left(d_{\mathrm{b}}-i_{\mathrm{al}}\right)^{2}$ and $\left(h_{\mathrm{b}}-j_{\mathrm{ab}}\right)^{2}$ thus reversing the direction of the bias to these components and to the dominance ratio. As heterosis may be observed for either of the above situations (where $A$ or $a$ is fixed in the background), it therefore follows that, at least in theory, the dominance ratio cannot provide a good indication as to whether its value will be high or low when heterosis is present. 


\section{Multiple loci}

When many pairs of loci show digenic interactions the overall impact of epistasis on the expression of heterosis is likely to be enormous, particularly when the level of epistasis is high. However, as the two-locus model represents the two extremes for gene dispersion and gene assciation, the situations represented by the multilocus case will fall well within the limits of the digenic model. Further, within the dimensions of the multilocus situation the impacts of ambidirectional dominance, unequal gene effects and various types of epistasis will have to be considered when interpreting heterosis.

The parameters which can be used to summarize the various kinds of interallelic and intergenic interactions have already been defined by Mather \& Jinks (1982) and therefore need not be elaborated further. The only difference is that the present situation complicates the interpretation of the $[i]$ and the $[j]$ component and thus reinforces the views of Mather \& Jinks (1982) that the signs and magnitudes of these components cannot be trusted for the determination of the type of epistasis prevailing in the material.

The impact of background genotype on the components of means and variances, however, has not been investigated before and therefore needs to be considered in detail. Assuming that a character is controlled by $K$ loci out of which $K_{1}$ are heterozygous and $K_{2}$ are fixed in a cross, contributions of these loci to the expectations of various generations will be as follows, where $d, h, i, j$ and $l$ and the various coefficients of association/dispersion ( $r d, r i$ and $r j$, etc.) have the same meaning and definitions as in Mather \& Jinks (1982).

(A) For the segregating loci:

overall mean $=m^{\prime}$;

additive deviation $[d]=r d_{1} \Sigma^{K_{1}} d$; dominance deviation $[h]=\Sigma^{K_{1}} h$; additive $\times$ additive interaction $[i]=r i_{1} \Sigma^{(1 / 2) K_{1}\left(K_{1}-1\right)} i$; additive $\times$ dominance interaction $[j]=r j_{1} \Sigma^{K_{1}\left(K_{1}-1\right)} j$; dominance $\times$ dominance interaction $[l]=\Sigma^{(1 / 2) K_{1}\left(K_{1}-1\right)} l$.

(B) For the background loci: additive deviation $[d]^{\prime}=r d_{2} \Sigma^{K_{2}} d$; additive $\times$ additive interaction $[i]^{\prime}=r i_{2} \Sigma^{(1 / 2) K_{2}\left(K_{2}-1\right)} i$; additive $\times$ additive interaction $[i]^{\prime \prime}=r i_{1} r i_{2} \Sigma^{K_{1} K_{2}} i$; additive $\times$ dominance interaction $[j]^{\prime}=r j_{2} \Sigma^{K_{1} K_{2}} j$.

Expectations given in Table 7 in terms of these components show that several components are confounded with each other and therefore cannot be estimated separately. As a matter of fact, when six parameters of the digenic interaction model are estimated in the presence of interactions between the segregating and the background loci, the various parameters account for the following effects:

$m=m^{\prime}+[d]^{\prime}+[i]^{\prime}$

$[d]=[d]+[i]^{\prime \prime}$

$[h]=[h]+[j]^{\prime}$

$[i]=[i]$;

$[j]=[j]$

$[l]=[l]$.

The background genotype can also have a marked impact on the variance components but only when the alleles of the background and segregating loci interact with each other. In a triple test cross analysis, these interactions affect the magnitudes of the additive and dominance components which then have the following expectations:

$D\left(=\sigma_{\mathrm{b}\left(\mathrm{F}_{\infty}\right)}^{2}\right)=\Sigma^{K_{1}}\left(d \pm \Sigma^{K_{2}} i\right)^{2}$ and

$H=\Sigma^{K_{1}}\left(h \pm \Sigma^{K_{2}} j\right)^{2}$ (lower sign for pairs in dispersion).

Thus, in addition to the biases due to interactions of the segregating loci, the estimates of $D$ and $H$ should

Table 7 Model accommodating the effects of the segregating and the background loci

\begin{tabular}{|c|c|c|c|c|c|c|c|c|c|c|}
\hline \multirow[b]{2}{*}{ Generation } & \multirow[b]{2}{*}{$m^{\prime}$} & \multicolumn{5}{|c|}{ Segregating loci } & \multicolumn{4}{|c|}{ Background loci } \\
\hline & & {$[d]$} & {$[h]$} & {$[i]$} & {$[j]$} & {$[l]$} & {$[d]^{\prime}$} & {$[i]^{\prime}$} & {$[i]^{\prime \prime}$} & {$[j]^{*}$} \\
\hline$P_{1}$ & 1 & 1 & 0 & 1 & 0 & 0 & 1 & 1 & 1 & 0 \\
\hline$P_{2}$ & 1 & -1 & 0 & 1 & 0 & 0 & 1 & 1 & -1 & 0 \\
\hline$F_{1}$ & 1 & 0 & 1 & 0 & 0 & 1 & 1 & 1 & 0 & 1 \\
\hline$F_{2}$ & 1 & 0 & 0.5 & 0 & 0 & 0.25 & 1 & 1 & 0 & 0.5 \\
\hline $\mathrm{F}_{3}$ & 1 & 0 & 0.25 & 0 & 0 & 0.0625 & 1 & 1 & 0 & 0.25 \\
\hline$F_{\infty}$ & 1 & 0 & 0 & 0 & 0 & 0 & 1 & 1 & 0 & 0 \\
\hline $\mathrm{B}_{1}$ & 1 & 0.5 & 0.5 & 0.25 & 0.25 & 0.25 & 1 & 1 & 0.5 & 0.5 \\
\hline $\mathrm{B}_{2}$ & 1 & -0.5 & 0.5 & 0.25 & -0.25 & 0.25 & 1 & 1 & -0.5 & 0.5 \\
\hline
\end{tabular}

*See text for definitions. 
also show the effect of epistasis which is attributable to background loci, whenever the latter are significant. The magnitude of this bias, however, will be proportionately smaller for $D$ (compared to $H$ ) unless the dominance ratio is high $(\sqrt{H / D} \geq 1)$ and the additive $\times$ additive and additive $\times$ dominance interactions show marked differences in their magnitudes or directions.

\section{Conclusion and implication}

The main conclusion from the study of interaction relationships is that both complementary and duplicate epistases supress heterosis even when the parents show gene dispersion (see Complementary and duplicate relationships, above). Similarly, heterosis is unlikely to be expressed in the presence of classical dominance epistasis under most circumstances. Only the classical recessive epistasis allows the dihybrid to perform better than its parents and even this happens only when the parents show gene dispersion. This implies clearly that many classical types of epsistasis do not contribute positively to heterosis, even when the magnitudes of the interaction components are larger than those of $d_{\mathrm{a}}$ and $h_{\mathrm{a}}$, etc.

There are, however, several other epistatic relationships that enhance the $F_{1}$ performance considerably (see Table 5). Further, as the effects of epistasis are not separated easily from those of the additive and dominance components, these relationships may generate spurious overdominance which may or may not be diagnosed correctly at the variance level. Perhaps the most important of these relationships are those that boost heterosis and also lead to the underestimation of dominance ratios because they are better able to explain the low values of dominance ratios that are observed frequently in most if not all the experiments (Kearsey \& Pooni, 1992; Pooni, 1994). A close scrutiny of these relationships, however, also shows that they cannot be responsible for heterosis in many real situations as they assume $[h]$ and $[l]$ to be positive (for positive heterosis), a situation which is rarely observed in practice.

Contributions of the background genotype to heterosis, on the other hand, are exclusively through its interactions with the segregating loci. Clearly, heterosis will be maximized when alleles at the segregating loci show a high degree of dispersion $\left(r i_{1} \simeq 0\right)$ while those at the background loci are mostly associated $\left(r_{2}>0\right.$; see Multiple loci, above). Further, the influence of these interactions is not restricted to the generation means alone and they also affect the dominance ratio which is likely to be inflated when there is positive heterosis and the true value of $\sqrt{H / D}$ is less than unity.
Another major impact of these interactions is on the distributions of the $F_{\infty}$ inbreds and the second cycle hybrids (Toledo et al., 1984) which become markedly non-normal in their presence. However, the examples of extreme non-normality are very rare, and consequently, it is perhaps not unrealistic to assume that background loci have only limited impact on the expression of genetic variation in general and heterosis in particular.

The present study has shown once again that gene dispersion and directional dominance (which may be partial or complete) are the true cause of heterosis. While modest levels of heterosis can be observed under most genetic situations, its exploitable magnitude nevertheless depends very much on the presence of dispersion and directional dominance. Epistasis and interactions between the background and the segregating loci may supplement this heterosis on many occasions. But being secondary sources of genetic variation they are not likely to be the sole cause of hybrid vigour, at least in a majority of the cases where the level of expressed heterosis is relatively high $(>30$ per cent $)$.

Finally, the present study has not revealed any model whereby $[h]$ and $[l]$ can be allowed to take opposite signs and contribute towards heterosis. As $[h]$ and $[l]$ are known to take opposing signs in a survey of experimental data (Kearsey \& Pooni, 1992), it therefore follows that either there are other relationships (between additive, dominance and epistatic effects of a pair of genes) that we have not identified or that epistasis actually supresses, or at least does not enhance, heterosis in many crop species (Pooni \& Jinks, 1981; Pooni, 1994).

\section{Acknowledgements}

The study was supported by a SERC studentship.

\section{References}

JINKS, J. L. 1954. The analysis of continuous variation in a diallel cross of Nicotiana rustica varieties. Genetics, 39, $767-788$.

JINKS, J. L. 1955. A survey of the genetical basis of heterosis in a variety of diallel crosses. Heredity, 9, 223-238.

JINKS, J. L. 1983. Biometrical genetics of heterosis. In: Frankel, R. (ed.) Heterosis. Monographs on Theoretical and Applied Genetics, pp. 1-46. Springer, Berlin.

KEARSEY, M. J. AND JINKS, J. L. 1968. A general method of detecting additive, dominance and epistatic variation for metrical traits. I. Theory. Heredity, 23, 403-409.

KEARSEY, M. J. AND POONI, H. S. 1992. The potential of inbred lines in the presence of heterosis. In: Dattee, Y., Dumas, C. and Gallais, A. (eds) Reproductive Biology and Plant Breeding, pp. 371-385. Springer, Berlin. 
MATHER, K. 1967. Complementary and duplicate gene interactions in biometrical genetics. Heredity, 27, 97-104.

MATHER, K. AND Jinks, J. L. 1982. Biometrical Genetics (3rd edn). Chapman and Hall, London.

POONI, H. S. 1976. Exploitation of inbreds in Nicotiana. Ph.D. thesis, University of Birmingham.

POONI, H. s. 1994. Genetics of heterosis and its implications for crop improvement. Ann. Biol., 9, 323-332.
POONI, H. S. AND JINKS, J. L. 1981. The true nature of non-allelic interactions in Nicotiana rustica revealed by association crosses. Heredity, 47, 253-258.

SMITH, H. H. AND ROBSON, D. S. 1959. Inheritance of dimensions of flower parts in tobacco. Proc. Int. Symp. on Biometrical Genetics, Ottawa. Pergamon, Oxford.

TOLEDO, J. F. F. DE, POONI, H. S. AND JINKS, J. L. 1984. Predicting the properties of second cycle hybrids produced by intercrossing random samples of recombinant inbred lines. Heredity, 53, 283-292. 